Oncology

\title{
A rare highly aggressive tumor of the kidney: The pure epithelioid angiomyolipoma
}

\author{
Mohamed Amine Bani ${ }^{\mathrm{a}, \mathrm{b}, *}$, Besma Laabidi ${ }^{\mathrm{a}, \mathrm{b}}$, Sarra Ben Rejeb ${ }^{\mathrm{a}, \mathrm{b}}$, Ramzi Khiari ${ }^{\mathrm{b}, \mathrm{c}}$, \\ Ammar Bouziani ${ }^{\mathrm{a}, \mathrm{b}}$, Issam Msakni ${ }^{\mathrm{a}, \mathrm{b}}$ \\ a Department of Pathology, Military Hospital, Tunis, Tunisia \\ ${ }^{\mathrm{b}}$ Faculté de Médecine de Tunis, Université Tunis El Manar, Tunis, Tunisia \\ ${ }^{\mathrm{c}}$ Department of Urology, Military Hospital, Tunis, Tunisia
}

\section{A R T I C L E IN F O}

Keywords:

Epithelioid variant

Renal angiomyolipoma

Renal neoplasm

Histology

Immunohistochemisty

\section{Introduction}

Renal epithelioid angiomyolipoma (EAML) has been recognized as a mesenchymal tumor in the 2016 World Health Organization classification of renal tumors, and a member of the family of PEComas, which may occur sporadically or in association with tuberous sclerosis syndrome (TSC). EAML is considered a potentially malignant neoplasm, since one third of the reported cases of EAML developed metastatic lesions. ${ }^{1}$ The "pure" form of EAML is extremely rare. It is composed of predominant or pure epithelial component and has minimal to no adipocytic component. ${ }^{2}$ We herin report a case of pure EAML of the kidney.

Case report

A 51 year-old man presented with complaint of a persistant mild pain in the left lumbar region. He denied hematuria, abdominal pain or recent illness. He denied personal or family history of genitourinary disease. Past medical history was not significant. The patient denied previous abdominal or genitourinary surgeries. Vital signs were within normal limits. Physical exam could not find any palpable lumbar mass or tenderness. Urinalysis showed no blood, leukocytes, or protein. A Renal ultrasound revealed a $12 \mathrm{~cm}$ hypoechoic mass within the upper pole of the left kidney. Serum tumor markers were within normal limits. Computed tomography (CT) of chest, abdomen and pelvis with intravenous contrast showed soft tissue mass of heterogeneous density, which measured $12 \times 10 \mathrm{~cm}$ and occupied the upper pole of the left kidney (Fig. 1). Scattered necrosis and calcification were occasionally observed, and the lesion exhibited moderate heterogeneous enhancement during enhanced scanning. Radiographically, the mass was considered to be renal cell carcinoma. No renal vein involvement was observed. It also showed an infiltration of the peri-renal fat and of the lombo-aortic nodes with no nodules in the liver, nor in the lung. The patient underwent a radical left nephrectomy. Gross pathology examination revealed an $11 \times 10 \times 8 \mathrm{~cm}$ whitish, non encapsulated mass with foci of necrosis and hemorrage. The mass invaded the renal sinus. The renal capsule was not invaded. No tumor thrombus in the renal vein was noted.

Routine Hematoxylin- and Eosin stained tissue sections revealed the mass to be composed of round pleomorphic and occasionally giant multinucleated tumor cells in a predominantly insular pattern (Fig. 2). Tumor cells had abundant eosinophilic cytoplasm, macronucleoli, and many atypical mitotic figures. There were few clear cells $(1 \%$ of the tumor). Some tumor cells had rhabdoid features. A lymphovascular invasion was demonstrated. There was focal necrosis ( $20 \%$ of the tumor volume). No adipous cells or smooth muscle cells were observed.

Immunohistochemical stains were performed, The tumor cells showed strong uniform cytoplasmic reactivity for Melan A (Fig. 3). The cells also showed a strong and patchy positivity for HMB-45 and a negativity for epithelial markers (epithelial membrane antigen, pancytokeratin) and Myogenin. The final diagnosis was pure epithelioid angiomyolipoma of the kidney.

\footnotetext{
* Corresponding author.

E-mail address: banimedamine@yahoo.fr (M.A. Bani).
} 


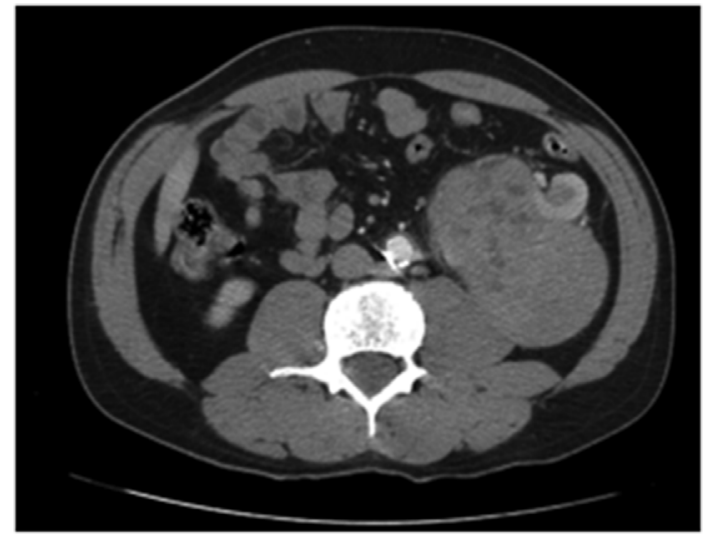

Fig. 1. Axial images of computed tomography scan showing $10 \mathrm{~cm}$ left upper pole mass.

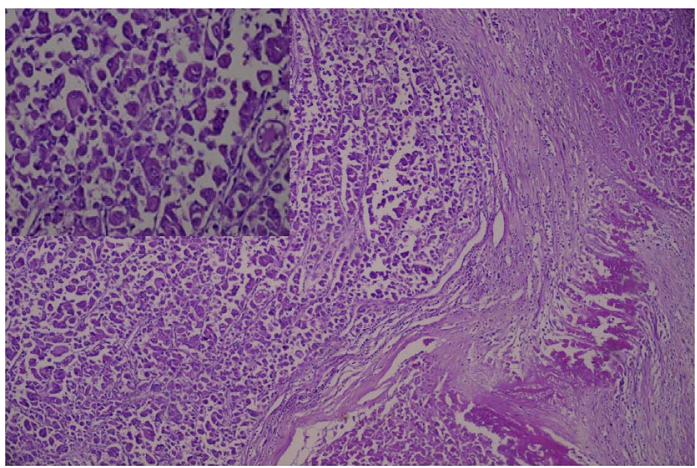

Fig. 2. Hematoxilin-Eosin $\times 100$ showed polyedric epitheloid proliferation in a primarily insular pattern with a large zone of necrosis. (Hematoxilin-Eosin $\times 400$ ) Tumor cells have abundant eosinophilic cytoplasm, distinct cell borders, and macronucleoli.

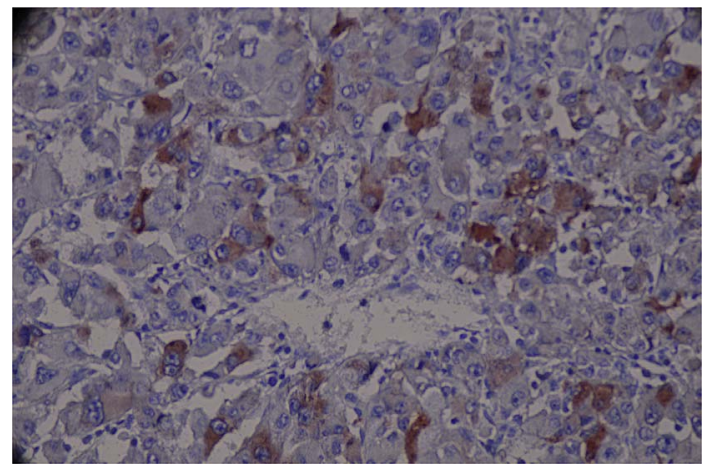

Fig. 3. Melan A staining at $400 \times$ magnification showed strong cytoplasmic staining.

Postoperative CT imaging conducted at six months of follow-up identified lung and left adrenal metastasis. In consequence, the patient was subjected to chemotherapy. The patient succumbed to neoplastic progression of the disease twelve months later.

\section{Discussion}

The epithelioid variant of renal angiomyolipoma is a rare entity. Its pure form, predominantly composed of epithelioid cells, is extremely rare. It occurs in $1 \%$ of renal AML. Its occurrence in association with TSC is higher than classic angiomyolipoma. ${ }^{1}$

Clinically, patients are frequently symptomatic, presenting with lumbar pain; some tumors are discovered during tuberous sclerosis follow-up. Our patient had no history of TSC and presented with a left lumbar pain. Imaging studies closely mimic renal cell carcinoma due to the low abundance of adipocytes in this tumor. ${ }^{1}$
Histologically classic angiomyolipoma has three components: mature adipose tissue, dysmorphic blood vessels, and spindle-shaped smooth muscle cells. In EAML, epithelioid cells are polygonal, atypical, with abundant cytoplasm, vesicular nuclei and prominent nucleoli. Some authors have suggested that at least $5 \%$ of cells must exhibit epithelioid histology to set the diagnosis of EAML. ${ }^{2}$ In our case, the tumor was totally composed of epithelioid cells.

Like in our case, EAML is usually positive for HMB45. However, some cases are negative for HMB45 and Melan-A, although electron microscopic examination of tumor cells confirmed the presence of premelanosome-like granules. ${ }^{1}$

Martignoni et al. first reported fatal cases of EAML in $1994 .^{3}$ In fact EAML metastases in one third of the cases. The liver and the lung are the most common sites of metastasis. Tumors with necrosis, mitotic activity, nuclear anaplasia, and external spread should raise significant concern for malignant outcome.

However, there are no histological criteria for malignant EAML, with the exception of distant metastases, which are accepted to be a definite sign of malignancy. Many recent studies examined histological parameters predictive of malignant behavior. Thus, Jun Lei et al. compared histological criteria in progressor and no progressor patients. A high percentage of epitheliod cells, marked atypia and large mass diameter were predictive of tumor progression. The 5 adverse prognostic parameters of Pure EAML were: tuberous sclerosis complex or concurrent angiomyolipoma, necrosis, tumor size $>7 \mathrm{~cm}$, extra-renal extension and/or renal vein involvement and carcinoma-like growth pattern. ${ }^{4}$ Recent studies also suggested that Ki67 index exceeding $10 \%$ and P53 overexpression and mutations in EAML may indicate the malignant behavior of the tumor. ${ }^{5}$

In our case, necrosis, high mitosis index, marked atypia, vascular involvement, and predominant epitheliod cells component were predictive of tumor progression. In fact, our patient had lung metastasis six months after surgery and died of tumor progression one year later. With clinical, imaging, gross examination, histological parameters and the presence or not of extra-renal extension, a prognostic classification of EAML into benign, potentially malignant, and malignant is possible. The former two are treated as classic AML. Malignant EAMLs are treated as a clear cell carcinoma. ${ }^{1}$

\section{Conclusion}

EAML is a rare tumor of the kidney characterised by epitheliod cells component and an expression of pre-melanocytic markers. Its pure form is extremely rare. When diagnosed, the risk of tumor progression is high, especially if other histological predictive parameters of progression are observed. In that case, active treatment should be undergone and patients should be closely monitored.

\section{Conflicts of interest}

None.

\section{References}

1. Martignoni G. Epithelioid angiomyolipoma. In: Eble JN, Sauter G, Epstein JI, Sesterhenn IA, eds. WHO Classification of Tumours of the Urinary System and Male Genital Organs. Lyon, France: IARC Press; 2016:65-66.

2. Jayaprakash PG, Mathews S, Azariah MB, Babu G. Pure epitheliod perivascular epitheloid cell tumor (epitheliod angiomyolipoma) of kidney: case report and literature review. J Canc Res Therapeut. 2014 Apr-Jun;10(2):404-406.

3. Martignoni G, et al. Renal epithelioid oxyphilic neoplasm (REON): a pleomorphic monophasic variant of renal angiomyolipoma. Int J Surg Pathol. 1994;2:539.

4. Lei JH, Liu LR, Wei Q, et al. A four-year follow-up study of renal epithelioid angiomyolipoma: a multi-center experience and literature review. Sci Rep. 2015 May $5 ; 5: 10030$.

5. Li W, Guo L, Bi X, Ma J, Zheng S. Immunohistochemistry of p53 and Ki-67 and p53 mutation analysis in renal epithelioid angiomyolipoma. Int J Clin Exp Pathol. 2015;8(8):9446-9451. 